\title{
Controls In Experimental Physics
}

\author{
A. Daneels
}

\author{
CERN, Geneva (Chairman EPCS) \\ Associate Member of EPS
}

The continuous progress made by data processing technology and the dramatic decrease in the cost of its hardware allow physicists to imagine evermore sophisticated machines to help break through physics barriers.

As in many industrial processes, experimental physics facilities are today unthinkable without a fleet of computers to ensure their smooth running. Particle accelerators for high energy physics and telescopes for astronomy illustrate research areas where many recent discoveries are strongly indebted to computer controls.

\section{Particle accelerators}

CERN's $800 \mathrm{MeV}$ booster synchrotron (Fig. 1) built in the early 1970's was the organization's first machine to rely to a major extent on computer controls. It had 2500 control channels accessible from a centralised computer system based on an IBM 1800 computer, with a satellite computer to drive the console display [1]. The machine was operated through a single dedicated console on which 30 application programs could be activated. Setting the operating conditions of the various components of the injection and transfer lines (particles are transferred from the injector into the main ring), such as the field strengths of electromagnets to steer and focus the beam and the frequency of RF cavities used to accelerate the particles, was carried out via simple, dedicated, computer-driven control panels.

Only a decade later, the controls of the very same machine employed at least five 512 Kbyte minicomputers, around 30 microcomputers each of 32 Kbyte and some 300 application programs [2]. The quantities of components such as power converters, timers, vacuum pumps, instruments for monitoring beam parameters, etc. had not increased significantly; more functions had instead been introduced so the

Axel Daneels, Chairman and a founder of the EPCS Group, is a Group Leader at CERN, Geneva, where he is responsible for software engineering in the Accelerator Technology Division. He studied electro-mechanical engineering at Ghent State University, Belgium and has had working visits to LANL, BNL and KEK.

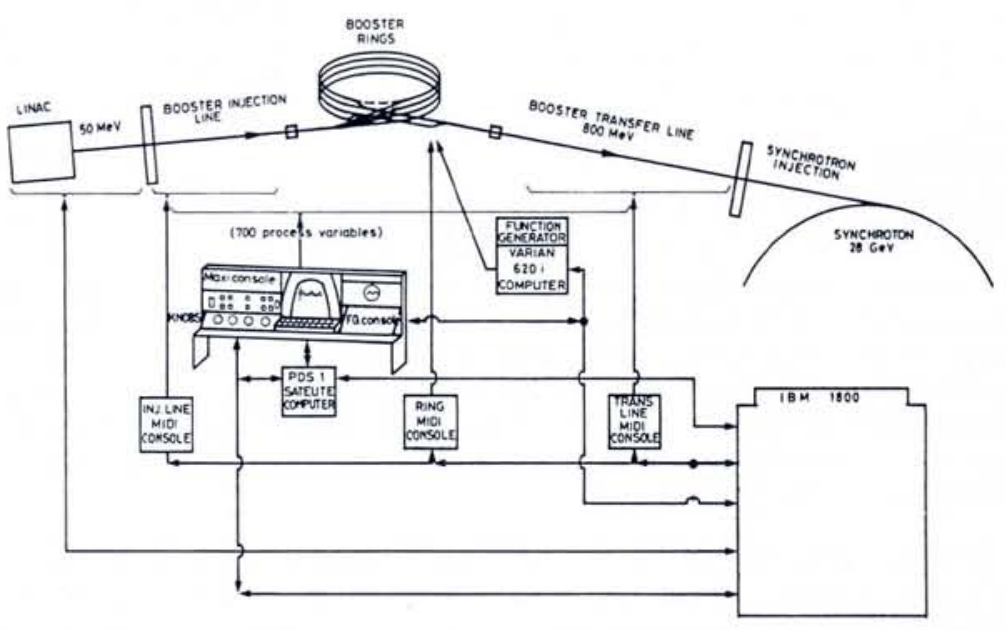

Fig. 1 - Controls for CERN's $800 \mathrm{MeV}$ booster ring completed in the mid-1970's. The four booster rings shown at the top combine single bunches of protons from the $50 \mathrm{MeV}$ linac into a single bunch with four times the intensity before they are injected into the $28 \mathrm{GeV}$ proton synchrotron (PS). One IBM 1800 computer with a "single user" open system was partitioned such that it interfaced to 3 midi-consoles and one maxi-console. The midi-consoles for controlling the injector line, booster rings and the transfer line were implemented for everyday operation. The maxi-console was set aside for more sophisticated controls and for complex beam measurements.

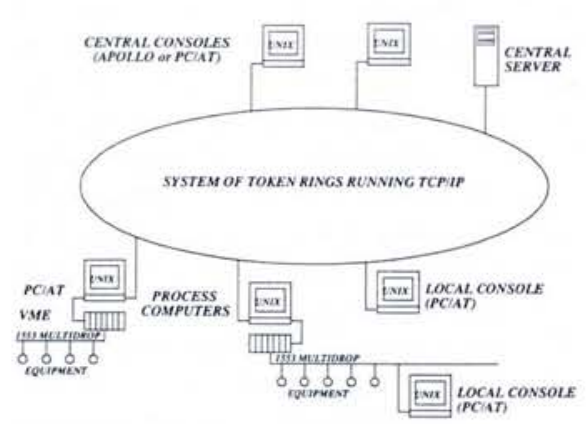

control channels now numbered 10000 .

But a true "explosion" eventually arose more from the operational facilities that were requested than from an increasing number of control channels, with a proportional increase in processing power. The $800 \mathrm{MeV}$ booster, with in its wake the entire $28 \mathrm{GeV}$ accelerator complex, was modified to produce beams of different particles and different characteristics during each of its one second cycles. Hundreds of devices had to be set every second and within a delay of $30 \mathrm{~ms}$ to working conditions specific to each beam. In addition, any console in the main control room had to permit the safe, simultaneous operation of any beam in any part of the machine
Fig. 2 - LEP's control architecture as commissioned in 1989 [3]: a system of token rings running parallel to the accelerator ring links workstations (PC - personal computers; process computers) connected to microprocessors (the vertically hatched rectangles) via a communications bus constructed complying to VME norms (see page 40). The microprocessors drive individual items of equipment via a so-called multidrop communications bus that conforms to another standard (MIL-STD-1553B).

yet still enable individual devices to be controlled from remote equipment rooms for test and maintenance purposes. The system thus satisfied the needs of a diverse clientele that included physicists, engineers, device specialists, computer scientists and machine operators. These requirements and the flexibility could not have been met without computers.

Newer controls, e.g. for CERN's LEP accelerator (Fig. 2), went one step further towards fully distributed systems with local intelligence at the device level - a must when equipment is spread over a large area (a ring $27 \mathrm{~km}$ in circumference) - by building on the next generation of data processing techno- 
LA SILLA

$2.2 \mathrm{~m}$ TELESCOPE

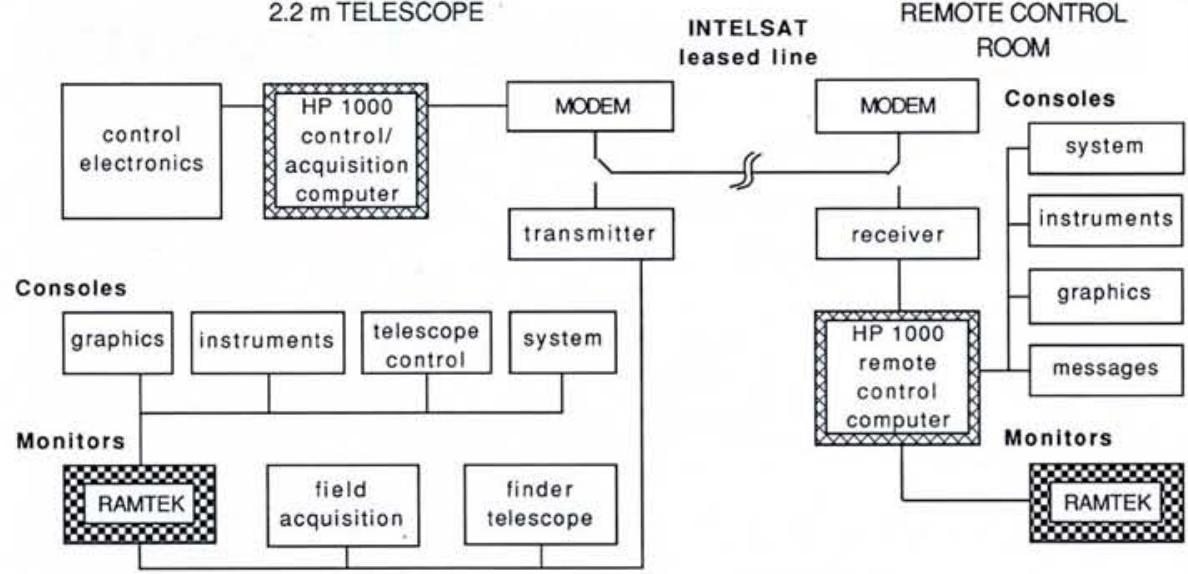

Fig 3 - Remote control of ESO's La Silla 2.2 m telescope from Garching: a schematic of the system configuration showing how instructions from consoles in Garching's Remote Control Room are relayed via a satellite link to La Silla's HP1000 computer that drives the telescope's electronic controls. Manually switching the link allows analogue images from the telescope's monitors to be observed in Garching on displays. Digital data acquired from the telescope's detectors by the HP1000 computer are transmitted to Garching by timesharing the link (G. Raffi, 1990).

logy. Broadly speaking, LEP's control architecture is based on networks at two different levels: there is a backbone (token ring or Ethernet) linking workstations that are in turn mostly connected to front-end microprocessors, or concentrators, which drive local distribution networks to individual devices.

\section{Telescopes}

Turning to astronomy and telescopes, the European Southern Observatory (ESO) has developed highly sophisticated observing methods that draw heavily upon computer controls. Two of the organization's telescopes in La Silla, Chile are operated via a satellite link from the ESO headquarters in Garching, Germany [4]. An astronomer can directly control each of the telescopes from the Remote Observation Room and set up instrumentation by inserting filters and diaphragms: data are read into the telescopes' computers and shipped, again via satellite, to another computer at the headquarters at the end of an observation session (Fig. 3).

ESO's new facility, the Very Large Telescope (VLT), will have mirrors of $8 \mathrm{~m}$, a size never conceived before. They will be thin, light-weight and flexible, their shape being adjusted using jacks. The feasibility of this technology (called active optics) has been demonstrated on the New Technology Telescope (NTT) that is now in operation. By applying computerised closed loop control it will be possible to observe on earth objects at nearly the same resolution as in space (adaptive optics [5]), so once again computer controls will open new horizons while yielding significant savings in investment costs.

\section{Engineered Control Systems}

It is no surprise that whereas in the 1970 's the cost of the control system for a major experimental facility in physics came to $5-10 \%$ of the total, it now approaches $20 \%$. Figures exceeding 100 man-years and multiples of 10 MECU are commonplace - amounts that are not unique to physics as even larger ones arise in industry. This growth obviously conflicts with the climate of economy that prevails in most laboratories so the control specialist has to evolve from being an all-round engineer or physicist who tinkered with an ad hoc system to become a member of a professional and organized team. The emphasis is now on engineering: control systems must represent managed economic trade-offs in the same way as most large engineering projects. Examples exist of control projects running out of, dare I say it, control by failing to fulfill their requirements because engineering was neglected. They often entailed wasteful redesign. Inversely, goals were met and significant savings realized when engineering principles were applied.

Control systems need to be designed not only to control a process but also, and to an equal extent, to maximize reliability and to ease development, integration and enhancement. Maintenance tends to consume significant resources and precious operation time so it must be taken into consideration at the design stage, together with diagnostic facilities. All of this requires software which takes the lion's share of modern systems, in contrast to hardware items which are now largely standardized. Managing the development is para- mount since projects should be based on proper cost estimates, solid planning with agreed priorities, realistic schedules, defined milestones and appropriate budget and resource allocations.

\section{The EPS Control Systems Group}

As increasing expertise is required in almost all domains of information technology (hardware interface, manmachine interface, communication, programming techniques, languages, operating systems, etc.) controls specialists need to exchange information and experience. Scarcer resources provide a strong motivation to work side by side in research or on the more straightforward development projects. This is precisely where the European Physical Society's Interdivisional Group on Experimental Physics Control Systems (EPCS) plays a rôle. It provides a forum for control specialists involved in experimental physics to exchange information, share experience and initiate joint projects. Some information describing the Group - its members, activities, organization and contacts - is given overleaf.

\section{International conferences}

Since its foundation in March 1986, the Group has sponsored two major international conferences (in Villars-surOllon, Switzerland [6] in 1987 with 180 participants and in Vancouver, Canada [3] in 1989 with 270 participants and a large number of industrial exhibitors). Shin-Ichi Kurokawa is currently working on the third conference that is to be held in the autumn of 1991 in Tsukuba, Japan where it will be hosted by KEK.

\section{Workshops and seminars}

The Group's first workshop took place in Grenoble at the European Synchrotron Radiation Facility (ESRF) in 1989. Specialists in controls discussed the possibility of developing a tool-kit, of the type now common in industry, for the implementation of computer applications programs for accelerator controls. The adoption of commercially available CASE tools for the computerassisted software engineering for the exchange of system designs or of entire packages was analyzed. Such tools would facilitate collaboration on large software projects arising in major physics experiments, e.g. high energy physics detectors. Follow-on seminars were held at CERN in 1989-90 to give representatives from about 15 laboratories unfamiliar with the methodologies some insights into the possibilities. Closed sessions to negotiate licensing policy and other financial matters with 
vendors of specific CASE tools followed technical presentations and product demonstrations. They resulted in vendors proposing a revised policy towards experimental physics laboratories and offering particularly favourable contractual conditions for EPCS members.

A workshop on real-time systems for microprocessors was organized by J.F. Gournay (CEN, Saclay) and T.T. Luong (GANIL, Caen) in Chamonix in January 1989. Currently available real-time systems were surveyed, in particular those that are popular for experimental physics control systems. Vendors were able to reconcile their more commercially oriented views with those of customers involved in an advanced technical field. The workshop, which was attended by representatives from 15 institutes, showed that laboratories are slowly converging towards a reduced spectrum of systems, and that software developers are making a real effort to offer compatible products. A follow-on workshop is being planned and it will be held jointly with the European Standardization Organization for Nuclear Electronics (ESONE).

\section{Working groups}

EPCS has also set up a Controls Protocols Committee chaired by G. Baribaud (CERN) to define standard protocols for operating different families of devices. Working groups chaired by G. Baribaud and G.P. Benincasa (CERN) are looking into protocols for power converters and beam instrumentation used in particle accelerators. The issues at stake have a strong economic impact as they involve standard interfaces, standard and hence more reliable software, clear separation of responsibilities between controls specialists and device specialists, efficient maintenance, etc.

A model describing the behaviour of power converters, an essential first step, has been agreed upon. Despite the variety of instrumentation that exists around accelerators, the second group has reached the promising conclusion that it might be possible to define a common protocol for many of the items. The Group will try to validate their protocol in several practical situations once its implementation at CERN has been shown to be a success.

\section{Projects}

The EPCS has loaned to the Joint European Torus (JET) workstations donated by Digital Equipment and Hewlett Packard to study advanced control systems for plasma fusion tokamak devices. The main outcome was that
JET has been able to decide upon a new operating environment (see page 26).

\section{Conclusions}

in view of the enormous cost of developing software, and the evolution of commercially available hardware and software products, it is essential that the EPCS Group provides a forum for discussions and related activities aimed at specifying requirements for standardized software programs and tools used in control applications in physics.

\section{REFERENCES}

[1] Baribaud G. et al., Proc. 9th Int. Conf. on High Energy Accelerators, Stanford, USA (1974)

[2] Baribaud G. et al., IEEE Trans. NS-28 (1981) 2267.

[3] Innocenti P.G., Nucl. Inst. and Methods A293 (1990) 1.

[4] Raffi G. and Ziebell M., Proc. of ESO's VLT Workshop, Venice, Italy (1986).

[5] Merkle F., The ESO Messenger, No. 60 (1990).

[6] Proc. Europhysics Conf. on Control Systems for Experimental Physics, CERN Report 90-08, Ed. B. Kuiper (1990).

\section{EPS Interdivisional Group EXPERIMENTAL PHYSICS CONTROL SYSTEMS}

EPCS was founded in 1986 under the auspices of the European Physical Society to provide a forum where controls specialists involved in experimental physics can exchange information, share experience, and initiate studies and collaborative projects in specific technical fields. The Group organizes conferences, workshops and seminars; works to define standard protocols through working groups; operates projects.

The Group is administered by a Board which meets twice each year. Official contact persons and the Chairman of working groups are invited to discuss activities at open sessions during the Board Meetings. Further information can be obtained from the Chairman or from the contact persons.

$$
\begin{aligned}
\text { Chairman: A. Daneels } & \\
& \text { CERN } \\
& \text { CH-1211 Geneva 23. } \\
& ++41(22) 7672581 \text { / } 7830221 \\
& \text { daneels@ cervm.cern.ch } \\
\text { Treasurer: } & \text { K. Müller } \\
& \text { KFA Jülich } \\
& \text { ZEL/NE } \\
& \text { Postfach 1913 } \\
& \text { W-5170 Jülich. } \\
& ++49 \text { (2461) } 616521 / 612525 \\
& \text { muller@djukfa52 }
\end{aligned}
$$

Board Members: T. Blumer, PSI

W. Busse, HMI

P.N. Clout, Vista, USA

B. Kuiper, CERN

E.C. Owen, Daresbury Lab.

\section{Member Institutes}

The EPCS has 39 institutes as members and each is represented by an official contact person(s), nominated by the Directorate of the institute, whose name(s) are listed below.

\section{Europe}

Centre d'Etude Nucléaire (CEN), Saclay: J.-F. Gournay

Centre de Recherche Nucléaire (CRN), Strasbourg: J. R. Lutz

Centre de Recherche en Physique des Plasmas (CRPP), Lausanne: J. Lister

Czech Technical University (CTU), Praha:

Y. Navratil

Daresbury Laboratory, Warrington: E.C. Owen

Dortmund Univ., Physics Dept.: C. Biermann

European Organization for High Energy

Physics (CERN), Geneva:

B. Kuiper, M. Rabany

European Southern Observatory (ESO),

Garching: G. Raff
European Synchrotron Radiation Facility (ESRF), Grenoble: W.D. Klotz

Forschungszentrum (KFA), Jülich:

U. Hacker

Gesellschaft für Schwerionenforschung (GSI), Darmstadt: R. Steiner

Grand Accélérateur National d'lons Lourds (GANIL), Caen: T.T. Luong

Hahn-Meitner-Institut (HMI), Berlin: W. Busse Institute for High Energy Physics (IHEP), Serpukhov: A. Dunaitsev

Joint European Torus (JET), Abingdon:

H. van der Beken

Laboratoire National Saturne, Gif-sur-Yvette: J.-P. Lagniel

Laboratori Nazionali Frascati (LNF), Frascati: L. Trasatti

Nationaal Instituut voor Kernfysica en Hoge Energie Fysica (NIKHEF), Amsterdam: W. Heubers

Next European Torus (NET)

Garching-bei-München: M. Browne

Paul Scherrer Institute (PSI), Villigen: T. Blumer

Rutherford Appleton Laboratory (RAL) Chilton: R.P. Mannix

Sincrotrone, Trieste: M. Mignacco

Africa

National Accelerator Centre (NAC).

Faure, RSA: F. Weehuizen

America

Argonne National Laboratory (ANL), Argone, IL: M. Knott

Brookhaven National Laboratory (BNL), Upton, NY: D. Barton

Continuous Electron Beam Accelerator Facility (CEBAF), Newport News, VA: R. Rossmith

Fermi National Accelerator Laboratory (FNAL), Batavia, IL: P. Lucas

Los Alamos National Laboratory (LANL), Los Alamos, NM: M. Thuot

Stanford Linear Accelerator Centre (SLAC). Stanford, CA: M. Ross

Superconducting Super Collider Laboratory Ellis County, TX: D.P. Gurd

TRIUMF, Vancouver, BC

Vista Control Systems, Inc., Los Alamos, NM: P.N. Clout

Asia

Bhabha Atomic Research Centre (BARC), Calcutta: S. Dasgupta

Heifei National Synchrotron Radiation

Laboratory (HESYRL), Heifei: C.Y. Yao

PRC Institute of High Energy Physics (IHEP), Beijing: S. Liu

Institute for Nuclear Physics (INP), Novosibirsk: G. Piskunov

Japan Atomic Energy Research Institute, JAERI: T. Kimura

National Laboratory for High Energy Physics (KEK), Tsukuba: S.-I. Kurokawa

Pohang Institute of Science and Technology, Pohang: S.C. Won 